\title{
Real-World Clinical Efficacy and Tolerability of Direct-Acting Antivirals in Hepatitis C Monoinfection Compared to Hepatitis C/Human Immunodeficiency Virus Coinfection in a Community Care Setting
}

Vijay Gayam ${ }^{1}$, Muhammad Rajib Hossain ${ }^{1}$, Mazin Khalid $^{1}$, Sandipan Chakaraborty ${ }^{1}$, Osama Mukhtar ${ }^{1}$, Sumit Dahal ${ }^{1}$, Amrendra Kumar Mandal ${ }^{1}$, Arshpal Gill ${ }^{1}$, Pavani Garlapati ${ }^{1}$, Sreedevi Ramakrishnaiah ${ }^{1}$, Khalid Mowyad ${ }^{2}$, Jagannath Sherigar ${ }^{3}$, Mohammed Mansour ${ }^{1}$, and Smruti Mohanty ${ }^{3}$

${ }^{1}$ Department of Medicine and Gastroenterology, Interfaith Medical Center, New York, NY, ${ }^{2}$ Department of Medicine, Detroit Medical Center, Wayne State University, Detroit, MI, and ${ }^{3}$ Division of Gastroenterology and Hepatology, Department of Medicine, New York-Presbyterian Brooklyn Methodist Hospital, New York, NY, USA

See editorial on page 609.

Background/Aims: Limited data exist comparing the safety and efficacy of direct-acting antivirals (DAAs) in hepatitis $\mathrm{C}$ virus $(\mathrm{HCV})$ monoinfected and $\mathrm{HCV} /$ human immunodeficiency virus (HIV) coinfected patients in the real-world clinic practice setting. Methods: All HCV monoinfected and HCV/HIV coinfected patients treated with DAAs between January 2014 and October 2017 in community clinic settings were retrospectively analyzed. Pretreatment baseline patient characteristics, treatment efficacy, factors affecting sustained virologic response at 12 weeks (SVR12) after treatment, and adverse reactions were compared between the groups. Results: A total of 327 patients were included in the study, of which 253 were HCV monoinfected, and 74 were HCV/HIV coinfected. There was a statistically significant difference observed in SVR12 when comparing HCV monoinfection and HCV/HIV coinfection ( $94 \%$ and $84 \%$, respectively, $p=0.005$ ). However, there were no significant factors identified as a predictor of a reduced response. The most common adverse effect was fatigue (27\%). No significant drug interaction was observed between DAA and antiretroviral therapy. None of the patients discontinued the treatment due to adverse events. Conclusions: In a real-world setting, DAA regimens have lower SVR12 in HCV/HIV coinfection than in HCV monoinfection. Further studies involving a higher number of HCV/HIV coinfected patients are needed to identify real predictors of a reduced response. (Gut Liver 2018;12:694-703)
Key Words: Hepatitis C, chronic; Direct acting antiviral agents; Sustained Virologic response; HCV/HIV coinfection

\section{INTRODUCTION}

Globally, an estimated 4 million to 5 million persons are chronically infected with both human immunodeficiency virus type 1 (HIV-1) and hepatitis C virus (HCV). ${ }^{1}$ One large acquired immune deficiency syndrome (AIDS) cohort, the EuroSIDA study, showed that there was positivity of anti-HCV antibody or HCV RNA positivity in approximately one-third of the cohort, emphasizing the importance of the HCV/HIV coinfected population. ${ }^{2} \mathrm{HCV}$ infection in HIV-positive patients results in a more aggressive liver disease with advanced fibrosis and earlier progression to end-stage liver disease. ${ }^{3-5}$ As a result, the importance of HCV eradication in HIV population is multifold and is associated with delayed progression of liver fibrosis, prevention of hepatocellular carcinoma and improved morbidity and mortality outcomes. ${ }^{6-8}$ Additionally, HCV treatment shows reduced liver injury from antiretroviral therapy (ART). ${ }^{9}$ A recent metaanalysis of HCV treated patients who have been on ART found that in addition to maintenance of HIV viral suppression there is a small rise in CD4 count compared with HIV monoinfected patients. ${ }^{10} \mathrm{HCV} / \mathrm{HIV}$ coinfected patients treated with interferonbased regimens are associated with significant drug interactions with ART and also had limited efficacy. ${ }^{11,12}$

Current guidelines indicate directly acting interferon-free oral antiviral regimens as the therapy of choice for both HCV and HCV/HIV coinfection. These agents target one of the nonstructural proteins-NS3/4A protease, NS5B polymerase and

Correspondence to: Vijay Gayam

Department of Medicine and Gastroenterology, Interfaith Medical Center, 1545 Atlantic Ave, Brooklyn, New York, NY 11213, USA

Tel: +1-718-613-4063, Fax: +1-718-780-4893, E-mail: vgayam@interfaithmedical.com

Received on December 29, 2017. Revised on February 27, 2018. Accepted on March 26, 2018. Published online September 28, 2018 pISSN 1976-2283 eISSN 2005-1212 https://doi.org/10.5009/gnl18004

() This is an Open Access article distributed under the terms of the Creative Commons Attribution Non-Commercial License (http://creativecommons.org/licenses/by-nc/4.0) which permits unrestricted non-commercial use, distribution, and reproduction in any medium, provided the original work is properly cited. 
the NS5A protein-critically involved in HCV replication. They are well tolerated, safe, and highly efficacious, and also negate the host factors like race, ethnicity and IL28B genotype from influencing sustained virologic response (SVR). Clinical trials have shown comparable efficacy with direct-acting antiviral (DAA) agents in both HCV monoinfection and HCV/HIV coinfection. ${ }^{13-16}$ However, significant drug interaction between DAA and ART is a primary concern for therapy in HCV/HIV coinfected group. ${ }^{17-20}$ Furthermore, the efficacy and tolerability of DAA in $\mathrm{HCV} / \mathrm{HIV}$ coinfected patients compared to HCV monoinfected patients in a real-world community hospital setting remains less clear. Most trials in the literature describing coinfections include HIV patients with undetectable viral load, and it is unclear whether coinfected patients with quantifiable viral load respond to the same extent as that of the undetectable viral load. As a result, this study was designed to assess the safety, efficacy, and tolerability of DAAs in HCV/HIV coinfected patients, and compare the findings with HCV monoinfected patients as well as with results from other studies in the literature. We also assessed the factors influencing sustained virologic response among the study population, particularly in the black population which constitutes the majority of our cohort.

\section{MATERIALS AND METHODS}

The study protocol was approved by the Interfaith Medical Center and New York-Presbyterian Brooklyn Methodist Hospital Institutional Review Board (IRB) and the patients were recruited from two specialty clinics attached to the two large community hospitals: Interfaith Medical Center and New York-Presbyterian Brooklyn Methodist Hospital located within a $6.5 \mathrm{~km}$ radius.

\section{Patients}

A total of 350 patients with chronic HCV were treated with DAAs between January 2014 and July 2017 at two institutions. Twenty-three patients were excluded from the study for various reasons including insufficient documentation of viral load during the treatment and failure to follow-up after the end of treatment. None of the excluded patients discontinued the treatment due to adverse events associated with treatment medications.

All the 327 patients included in this retrospective cohort study received at least 12 weeks of treatment with one of the recommended combination regimens in standard doses for chronic HCV infection. Patients were divided into two groups: patients with HCV monoinfection $(\mathrm{n}=253)$ and patients with HIV and HCV coinfection $(n=74)$. Combination treatment regimens used were sofosbuvir+ribavirin (SOF+RBV), ledipasvir+sofosbuvir (LDV/SOF), ledipasvir+sofosbuvir+ribavirin (LDV/SOF+RBV), elbasvir+grazoprevir (EBR/GZR), sofosbuvir+velpatasvir (SOF/ VEL), ombitasvir+paritaprevir+ritonavir+dasabuvir (OBV/PTV/ $\mathrm{r}+\mathrm{DSV}$ ), ombitasvir+paritaprevir+ritonavir+dasabuvir+ribavirin $(\mathrm{OBV} / \mathrm{PTV} / \mathrm{r}+\mathrm{DSV}+\mathrm{RBV})$, daclatasvir+ribavirin $(\mathrm{DCV}+\mathrm{RBV})$ and simeprevir+sofosbuvir (SMV/SOF) (Fig. 1). Duration of treatment period ranged from 12 weeks $(n=291)$ to 24 weeks $(n=36)$ as per guideline depending on their status of prior treatment, viral load and cirrhosis.

\section{Study assessments}

Treatment safety and tolerability were assessed by reviewing patient's chart regarding adverse events, dose adjustment or discontinuation of medication and treatment completion rates. To determine lab abnormality related to antivirals used, pretreatment laboratory values were compared to post-treatment values. Most patients without clinical and laboratory evidence of cirrhosis were treated without any assessment for liver fibrosis. Similarly, patients were considered cirrhotic without further assessments for fibrosis when clinical, laboratory and radiologic evidence of cirrhosis were present. Wherever indicated, noninvasive tests like a FibroSure test or the FibroScore test and aspartate aminotransferase-to-platelet ratio (APRI) index score were mainly used to identify liver fibrosis and occasionally with liver biopsy. Treatment efficacy and tolerability were then compared between the monoinfected and coinfected groups.

Treatment response was assessed with HCV RNA viral load (IU/ $\mathrm{mL}$ ) at 4 weeks after initiation of treatment, at the end of treatment, and 12 weeks after completion of treatment. The test was performed using COBAS ${ }^{\circledR}$ AmpliPrep/COBAS ${ }^{\circledR}$ TaqMan $^{\circledR}$ HCV Quantitative Test, v2.0 (Roche Molecular Diagnostics, Basel, Switzerland) with the lower limit of quantification of HCV RNA

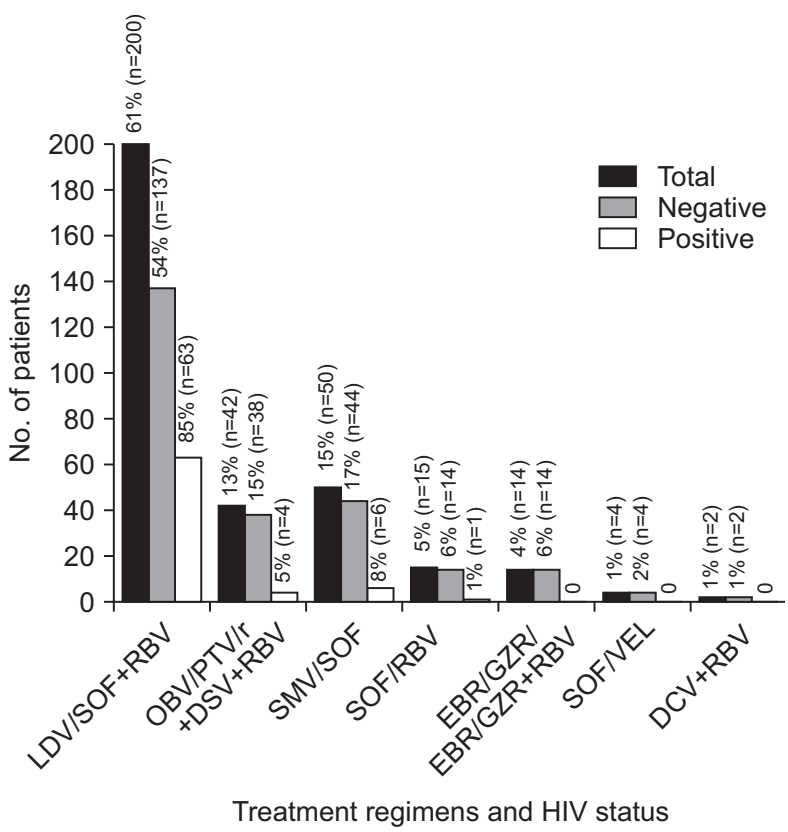

Fig. 1. Treatment regimens used and the number of patients in each patient group.

LDV, ledipasvir; SOF, sofosbuvir; RBV, ribavirin; OBV, ombitasvir; PTV/r, paritaprevir+ritonavir; DSV, dasabuvir; SMV, simeprevir; EBR, elbasvir; GZR, grazoprevir; VEL, velpatasvir; DCV, daclatasvir; HIV, human immunodeficiency virus. 
$15 \mathrm{IU} / \mathrm{mL}$. SVR12 was defined as the undetectable viral load at 12 weeks after the end of treatment. Failure was defined as the post-treatment relapse (detectable HCV RNA after the end of treatment or 12 weeks after completion of treatment), confirmed breakthrough (an increase from undetectable to quantifiable RNA level or at least $1 \log 10$ above nadir) during treatment, partial response, defined as patients who achieved a $2 \log 10$ drop in HCV RNA by week 12 of treatment, but did not achieve an end of treatment response or the presence of quantifiable HCV RNA that is not otherwise defined as breakthrough, partial response or relapse. Treatment efficacy and tolerability were compared between the monoinfected and coinfected groups.

\section{Statistical analysis}

Statistical analysis was done using SPSS statistics software package version 21.0 (IBM Corp., Armonk, NY, USA). Descriptive statistics were used for evaluation of initial patient's data including clinical, laboratory and demographic characteristics. Normally distributed values were expressed as the mean and standard deviation and mean quantitative values were analyzed using student t-test. SVR12 were expressed as percentages (\%). Chi-square test was applied as appropriate for analyzing differences in qualitative values. One-way analysis of variance was used to determine whether there were differences among the group means. Univariate was used for assessing factors related to SVR12. A p-value less than 0.05 was considered significant. Multivariable logistic regression was performed only in variables with a p-value $<0.05$ in univariate analysis.

\section{RESULTS}

\section{Baseline characteristics and treatment regimens used}

Three hundred and twenty-seven patients were included in the study (Table 1). Seventy-seven percent $(n=253)$ had HCV monoinfection and 23\% (n=74) had HCV/HIV coinfection. The mean age of patients in the study was $60.05 \pm 11.057$ years and was comparable in the HCV monoinfection and the HCV coinfection groups $(60.61 \pm 11.46$ years vs $58.11 \pm 9.35$ years $)$. Majority of the population in the HCV monoinfection and the HCV coinfection groups were male (60\% vs 65\%, respectively), black (64\% vs 66\%, respectively), and obese (38\% vs $30 \%$ ). Around $8 \%$ of the patients were HCV treatment naïve in both groups (79\% in HCV monoinfection vs 78\% in HCV/ HIV coinfection group, $\mathrm{p}=0.871$ ). Among the patients with HCV monoinfection, 53\% had genotype 1a and 25\% had genotype $1 \mathrm{~b}$ while among those with coinfection, 62\% had genotype $1 \mathrm{a}$ and $22 \%$ had genotype $1 \mathrm{~b}(\mathrm{p}=0.481)$. The monoinfection and coinfection groups were also comparable in terms of initial HCV viral load $(4,171,305.91 \pm 7,801,895.87 \mathrm{IU} / \mathrm{mL}$ vs $3,720,970.03 \pm 5,480,889.85 \mathrm{IU} / \mathrm{mL}, \mathrm{p}=0.678$ ), proportion of population with APRI score of 1 or more (29\% vs 35\%, p=0.390), proportion of population with compensated cirrhosis (23\% vs
$14 \%, \mathrm{p}=0.103)$. None of the patients had decompensated cirrhosis.

Treatment regimen varied among the cohort; $61 \%(n=200)$ of the patients were treated with LDV/SOF or LDV/SOF and ribavirin, 13\% $(\mathrm{n}=42)$ with $\mathrm{OBV} / \mathrm{PTV} / \mathrm{r}+\mathrm{DSV}$ or $\mathrm{OBV} / \mathrm{PTV} / \mathrm{r}+\mathrm{DSV}$ and ribavirin, $15 \%(n=50)$ with SMV+SOF, $0.5 \%(n=15)$ with SOF and ribavirin while the rest were treated with EBR/GZR, EBR/ GZR + ribavirin, SOF/VEL and DCV+ribavirin.

\section{Response to treatment}

The overall SVR in all patients observed in the study was $94 \%$. The univariate analysis determined the factors associated with the SVR and multivariate analysis was also performed on variables with significant findings (with $\mathrm{p}$-value $<0.05$ in univariate analysis) (Table 2). SVR was higher with DAA treatment in HCV monoinfection as compared to HCV/HIV coinfection (96\% vs 86\%) which was statistically significant, $p=0.005$ (Fig. 2). Even after adjusting baseline characteristics in multivariable logistic regression models, this finding was consistent ( $\mathrm{p}=0.005)$. SVR12 was 95\% in the LDV/SOF/LDV/SOF+ribavirin group,

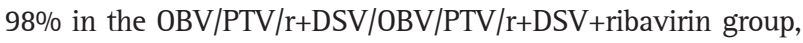
$88 \%$ in the SMV+SOF group, $80 \%$ in the SOF+ribavirin group and $100 \%$ in the EBR/GZR/EBR/GZR+ribavirin group as well as the SOF/VEL and DCV+ribavirin. In the overall cohort, there were no significant differences observed in SVR achievement between the two groups based on the sex, body mass index (BMI), APRI, Child-Turcotte-Pugh (CTP) score, age, race, HCV genotype, HCV viral load, prior HCV treatment status, baseline hemoglobin level, hepatic enzyme level, presence or absence of cirrhosis, or Model for End-Stage Liver Disease (MELD) score.

Univariate analysis of the factors associated with SVR (Table 3) showed that the patients who failed to achieve SVR12 as compared to those who did achieve SVR12 had higher mean pretreatment HIV viral load (90.78 IU/mL vs $62.84 \mathrm{IU} / \mathrm{mL}, \mathrm{p}=0.01)$, higher mean pretreatment HCV viral load $(4,512,134 \mathrm{IU} / \mathrm{mL}$ vs $3,434,891 \mathrm{IU} / \mathrm{mL}, \mathrm{p}<0.05$ ) and lower mean baseline CD4 count (458 cells/mL vs 610 cells/mL, $\mathrm{p}<0.05$ ). However, after adjusting variables and baseline characteristics in multivariate analysis, these findings were not consistent and it showed no difference in SVR achievement based on baseline HCV and HIV viral load, pretreatment CD4 count. SVR was higher in coinfected females than coinfected males ( $100 \%$ vs 79\%, $\mathrm{p}=0.012$ ) but again, multivariate analysis did not show any significant difference based on gender. Also, SVR in the coinfected cohort showed no statistically significant associations with age, race, BMI, HCV genotype, HCV prior treatment status, APRI score, MELD score, CTP class, presence or absence of compensated cirrhosis, baseline aspartate aminotransferase, baseline alanine aminotransferase, baseline hemoglobin or baseline platelet levels.

\section{Genotype and treatment outcome}

More than half of our patients were infected with HCV geno- 
Table 1. Baseline Characteristics of All 327 Patients

\begin{tabular}{|c|c|c|c|c|}
\hline \multirow{2}{*}{ Characteristics } & \multirow{2}{*}{$\begin{array}{c}\text { Total } \\
(\mathrm{n}=327)\end{array}$} & \multicolumn{2}{|c|}{ HIV status } & \multirow{2}{*}{ p-value } \\
\hline & & HIV negative ( $\mathrm{n}=253$ ) & HIV positive $(\mathrm{n}=74)$ & \\
\hline Age, yr & $60.05 \pm 11.057$ & $60.61 \pm 11.461$ & $58.11 \pm 9.358$ & 0.087 \\
\hline Sex & & & & 0.587 \\
\hline Male & $201(61.4)$ & $153(60.4)$ & $48(64.9)$ & \\
\hline Female & $126(38.5)$ & $100(39.5)$ & $26(35.1)$ & \\
\hline Race & & & & 0.272 \\
\hline White & $49(15)$ & 42 (16.6) & $7(9.4)$ & \\
\hline Black & $211(64.5)$ & $162(64)$ & $49(66.2)$ & \\
\hline Asian & $1(0.3)$ & $1(0.4)$ & $0(0)$ & \\
\hline Hispanic & $21(64.2)$ & $13(5.2)$ & $8(10.8)$ & \\
\hline Other & 45 (13.7) & 35 (13.8) & $10(13.5)$ & \\
\hline BMI, $\mathrm{kg} / \mathrm{m}^{2}$ & $28.323 \pm 5.5836$ & $28.479 \pm 5.5021$ & $27.792 \pm 5.8613$ & 0.353 \\
\hline BMI, $\mathrm{kg} / \mathrm{m}^{2}$ & & & & 0.217 \\
\hline$<30$ & 209 (63.9) & $157(62)$ & $52(70.2)$ & \\
\hline$\geq 30$ & $118(36.1)$ & 96 (37.9) & 22 (29.8) & \\
\hline Prior treatment & & & & 0.871 \\
\hline $\mathrm{TN}$ & 259 (79.2) & 201 (79.5) & $58(78.3)$ & \\
\hline $\mathrm{TE}$ & $68(20.85)$ & $52(20.5)$ & $16(21.4)$ & \\
\hline Genotype & & & & 0.481 \\
\hline $1 \mathrm{a}$ & $181(55.3)$ & $135(53.3)$ & $46(62.1)$ & \\
\hline $1 \mathrm{~b}$ & $80(24.4)$ & $64(25.2)$ & 16 (21.6) & \\
\hline 2 & $16(4.9)$ & $14(5.5)$ & $2(2.7)$ & \\
\hline 3 & $12(3.6)$ & $11(4.3)$ & $1(1.35)$ & \\
\hline 4 & 38 (11.6) & 29 (11.4) & $9(12.1)$ & \\
\hline Initial HCV viral load & $4,063,655.90 \pm 7,306,667.790$ & $4,171,305.91 \pm 7,801,895.875$ & $3,720,970.03 \pm 5,480,889.858$ & 0.678 \\
\hline Initial HIV viral load & - & - & $66.71 \pm 252.888$ & - \\
\hline Initial CD4 count & - & - & $589.25 \pm 307.636$ & - \\
\hline APRI score & & & & 0.390 \\
\hline$<1$ & $227(69.4)$ & $179(70.7)$ & $48(65)$ & \\
\hline$\geq 1$ & $100(30.5)$ & $74(29.2)$ & $26(35)$ & \\
\hline Cirrhosis & & & & 0.103 \\
\hline No & $260(79.5)$ & $196(77.5)$ & $64(86.4)$ & \\
\hline Yes & $67(20.5)$ & 57 (22.5) & $10(13.6)$ & \\
\hline MELD score & & & & 0.007 \\
\hline$<10$ & $239(73)$ & $194(77)$ & $45(60.8)$ & \\
\hline$\geq 10$ & $87(27)$ & $58(23)$ & 29 (39.2) & \\
\hline CTP class & & & & 0.062 \\
\hline A & $289(88.3)$ & $228(90.4)$ & $61(82.4)$ & \\
\hline $\mathrm{B}$ & $37(11.7)$ & $24(9.5)$ & $13(17.5)$ & \\
\hline \multicolumn{5}{|l|}{ Other comorbidities } \\
\hline Diabetes & $105(32.1)$ & 84 (33.2) & $21(28.3)$ & 0.481 \\
\hline Hypertension & $165(50.4)$ & $134(52.9)$ & $31(41.8)$ & 0.113 \\
\hline Coronary artery disease & $31(9.4)$ & $28(11)$ & $3(4)$ & 0.074 \\
\hline Chronic kidney disease & $25(7.6)$ & $19(7.5)$ & $6(8)$ & 0.808 \\
\hline End-stage renal disease & $2(0.6)$ & $1(0.3)$ & $1(1.3)$ & 0.402 \\
\hline Chronic anemia & $8(2.4)$ & $7(2.7)$ & $1(1.3)$ & 0.688 \\
\hline
\end{tabular}

Data are presented as mean \pm SD or number $(\%)$.

HIV, human immunodeficiency virus; BMI, body mass index; TN, treatment naïve; TE, treatment experienced; HCV, hepatitis C virus; APRI, aspartate aminotransferase-to-platelet ratio index; MELD, Model for End-Stage Liver Disease; CTP, Child-Turcotte-Pugh. 
Table 2. Factors Associated with SVR by Univariate Analysis and Multivariate Analysis

\begin{tabular}{|c|c|c|c|c|c|}
\hline & $\begin{array}{c}\text { Total } \\
(n=327)\end{array}$ & $\begin{array}{l}\text { Achieved SVR12 } \\
\qquad(\mathrm{n}=308)\end{array}$ & $\begin{array}{l}\text { Did not achieve SVR12 } \\
\qquad(\mathrm{n}=19)\end{array}$ & $\begin{array}{l}\text { Univariate } \\
\text { p-value }\end{array}$ & $\begin{array}{c}\text { Multivariate } \\
\text { p-value }\end{array}$ \\
\hline HIV status (positive/negative) & $253 / 74$ & $244 / 64$ & $9 / 10$ & 0.003 & 0.005 \\
\hline Sex (male/female) & $201 / 126$ & $186 / 122$ & $15 / 4$ & 0.145 & - \\
\hline Age, yr & $60.05 \pm 11.057$ & $60.01 \pm 10.937$ & $60.63 \pm 13.167$ & 0.812 & - \\
\hline Race (W/B/A/H/O) & $49 / 211 / 1 / 21 / 45$ & $46 / 198 / 1 / 20 / 43$ & $3 / 13 / 0 / 1 / 2$ & 0.989 & - \\
\hline BMI, $\mathrm{kg} / \mathrm{m}^{2}$ & $28.323 \pm 5.5836$ & $28.494 \pm 5.5957$ & $25.554 \pm 4.6906$ & 0.026 & 0.592 \\
\hline BMI $\left(<30 / \geq 30 \mathrm{~kg} / \mathrm{m}^{2}\right)$ & $209 / 118$ & $195 / 113$ & $14 / 5$ & 0.464 & - \\
\hline Prior treatment (TN/TE) & $259 / 68$ & $245 / 63$ & $14 / 5$ & 0.561 & - \\
\hline GT (1a/1b/2/3/4) & $181 / 80 / 16 / 12 / 38$ & $168 / 79 / 15 / 11 / 35$ & $13 / 1 / 1 / 1 / 3$ & 0.391 & - \\
\hline \multirow[t]{2}{*}{ HCV RNA, IU/mL } & $3,984,412.73 \pm$ & $3,980,781.64 \pm$ & $4,042,701.26 \pm$ & 0.970 & - \\
\hline & $6,913,685.42$ & $7,072,669.149$ & $3,591,177.899$ & & \\
\hline HIV RNA, IU/mL & $66.71 \pm 252.888$ & $62.84 \pm 260.328$ & $211.988 \pm 90.78$ & 0.761 & - \\
\hline CD4 count, cells/mL & $589.25 \pm 307.636$ & $610.30 \pm 308.668$ & $458.22 \pm 282.279$ & 0.170 & - \\
\hline APRI score $(<1 / \geq 1)$ & $227 / 100$ & $219 / 89$ & $8 / 11$ & 0.008 & 0.501 \\
\hline MELD score $(<10 / \geq 10)$ & $239 / 87$ & $229 / 78$ & $10 / 9$ & 0.057 & - \\
\hline CTP class (A/B) & $289 / 37$ & $276 / 31$ & $13 / 6$ & 0.013 & 0.208 \\
\hline Compensated cirrhosis (no/yes) & $260 / 67$ & $248 / 60$ & $12 / 7$ & 0.081 & - \\
\hline $\mathrm{ALT}, \mu / \mathrm{L}$ & $65.14 \pm 95.464$ & $64.98 \pm 97.213$ & $67.63 \pm 62.156$ & 0.907 & - \\
\hline $\mathrm{AST}, \mu / \mathrm{L}$ & $59.57 \pm 60.863$ & $58.84 \pm 61.538$ & $71.42 \pm 48.407$ & 0.814 & - \\
\hline $\operatorname{ALT}(<40 / \geq 40 \mu / \mathrm{L})$ & $138 / 189$ & $131 / 177$ & $7 / 12$ & 0.812 & - \\
\hline Hemoglobin, g/dL & $13.270 \pm 1.6411$ & $13.254 \pm 1.6600$ & $13.537 \pm 1.3044$ & 0.466 & - \\
\hline Platelets, $\mathrm{K} / \mu \mathrm{L}$ & $193.95 \pm 76.092$ & $197.16 \pm 74.160$ & $141.95 \pm 89.612$ & 0.002 & 0.124 \\
\hline
\end{tabular}

Data are presented as number or mean \pm SD.

SVR12, sustained virologic response at 12 weeks after treatment; HIV, human immunodeficiency virus; W, white; B, black; A, Asian; H, Hispanic; 0, others; BMI, body mass index; TN, treatment naïve; TE, treatment experienced; GT, genotype; HCV, hepatitis C virus; APRI, aspartate aminotransferase-to-platelet ratio index; MELD, Model for End-Stage Liver Disease; CTP, Child-Turcotte-Pugh; ALT, alanine aminotransferase; AST, aspartate aminotransferase.

*Only variables with a p-value $<0.05$ in a univariate analysis were assessed.

type 1a (55.3\%) and 24.5\% infected with genotype $1 \mathrm{~b}$. In the overall cohort, SVR was $92.8 \%$ in genotype 1a, 98.7\% in genotype 1 b, 93.7\% in genotype 2, 91.7\% in genotype 3 and 92.1\% in genotype 4. In the HCV/HIV coinfected population, SVR was $87 \%$ in genotype 1a, $93.7 \%$ in genotype $1 \mathrm{~b}, 100 \%$ in genotype 2, 100\% in genotype 3 and 66.7\% in genotype 4 . There was no statistically significant difference observed in SVR rates based on genotypes (Fig. 3).

\section{Prior treatment status and treatment failure}

Nearly a fifth of the patients $(n=68)$ had prior treatment for HCV. Overall SVR amongst those with prior treatment was $92.6 \%$ as compared to $94.6 \%$ in those who were treatment naïve ( $\mathrm{p}=0.561)$. Similarly, amongst the HCV-HIV coinfected patients, there was no difference in SVR between those with previous treatment and those without prior treatment (75\% vs $89.7 \%$, $\mathrm{p}=0.208$ ).

Among those with treatment failure, 11 had relapsed after the treatment, seven had a partial response and one had a breakthrough during the treatment. Drug resistance testing was not done in those patients making it difficult to identify the actual cause of relapse. Clinical characteristics of the 19 patients who did not achieve a SVR are shown in Table 4.

Tolerability and side effects: The most common adverse effects reported were fatigue (27\%), anemia (14\%), and leucopenia (11\%) (Table 5). Except for abdominal pain and leucopenia, the incidence of adverse effects was similar in the HCV monoinfection and HCV/HIV coinfection groups. None of the patients in our study required discontinuation or adjustment of medication dosage due to drug interaction or side effects. Medication compliance was as reported 100\%. (0.3\%)

\section{DISCUSSION}

In the post-interferon era, first-generation protease inhibitors telaprevir and boceprevir with ribavirin was the cornerstone of therapy and achieved an SVR up to 75\% in patients with HCV genotype $1 .^{21,22}$ The development of more efficient and tolerable antiviral agents, interferon-free second-generation DAA, is now the first line regimen as per current guidelines to fight HCV in- 


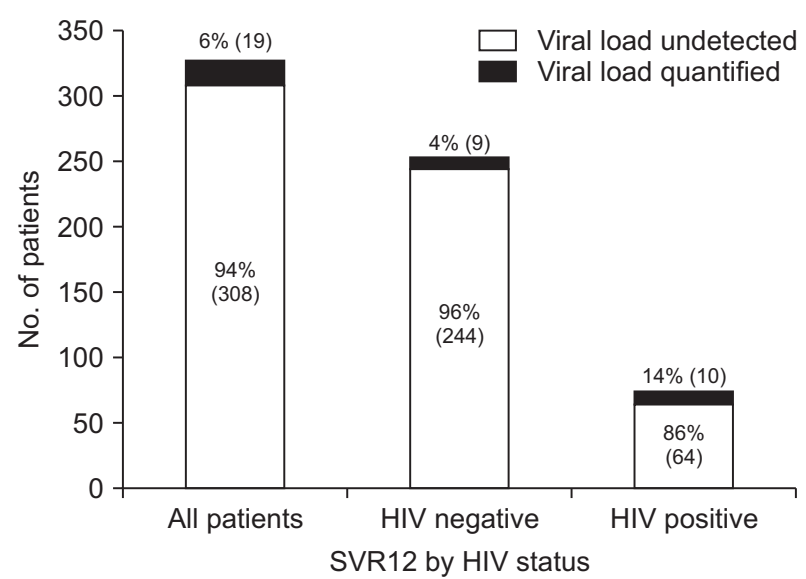

Fig. 2. Treatment response in each group measured by overall sustained virologic response at 12 weeks post-treatment (SVR12). HIV, human immunodeficiency virus.

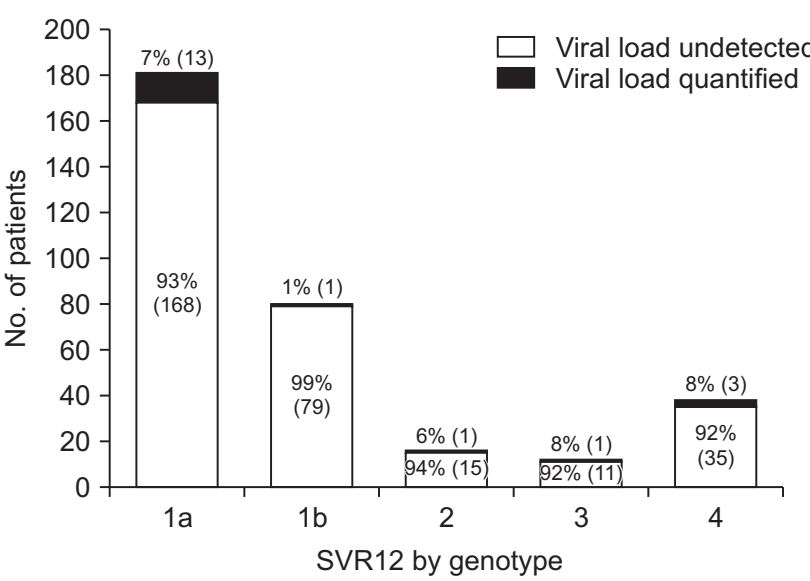

Fig. 3. Sustained virologic response at 12 weeks post-treatment (SVR12) by genotype.

Table 3. Univariate and Multivariate Analysis of Factors Associated with SVR in HCV-HIV Coinfected Patients

\begin{tabular}{|c|c|c|c|c|c|}
\hline & $\begin{array}{l}\text { Total } \\
(\mathrm{n}=74)\end{array}$ & $\begin{array}{l}\text { Achieved SVR12 } \\
\qquad(\mathrm{n}=64)\end{array}$ & $\begin{array}{l}\text { Did not achieve SVR12 } \\
\qquad(\mathrm{n}=10)\end{array}$ & $\begin{array}{l}\text { Univariate } \\
\text { p-value }\end{array}$ & $\begin{array}{c}\text { Multivariate } \\
\text { p-value* }\end{array}$ \\
\hline Sex (male/female) & $48 / 26$ & $38 / 26$ & $10 / 0$ & 0.012 & 0.96 \\
\hline Age, yr & $58.11 \pm 9.358$ & $58.13 \pm 9.221$ & $58.00 \pm 10.729$ & 0.225 & - \\
\hline Race (W/B/A/H/O) & $7 / 49 / 0 / 8 / 10$ & $6 / 42 / 0 / 8 / 8$ & $1 / 7 / 0 / 0 / 2$ & 0.651 & - \\
\hline BMI, yr & $27.792 \pm 5.8613$ & $28.119 \pm 5.9709$ & $25.700 \pm 4.8445$ & 0.307 & - \\
\hline BMI $\left(<30 / \geq 30 \mathrm{~kg} / \mathrm{m}^{2}\right)$ & $52 / 22$ & $45 / 19$ & $7 / 3$ & 0.623 & - \\
\hline Prior treatment (TN/TE) & $58 / 16$ & $52 / 12$ & $6 / 4$ & 0.208 & - \\
\hline GT $(1 \mathrm{a} / 1 \mathrm{~b} / 2 / 3 / 4)$ & $46 / 16 / 2 / 1 / 9$ & $40 / 15 / 2 / 1 / 6$ & $6 / 1 / 0 / 0 / 3$ & 0.376 & - \\
\hline \multirow[t]{2}{*}{ HCV RNA, IU/mL } & $3,582,459.44 \pm$ & $3,434,891.98 \pm$ & $4,512,134.40_{ \pm}$ & 0.000 & 0.25 \\
\hline & $5,100,958.57$ & $5,340,624.38$ & $3249,419.14$ & & \\
\hline HIV RNA, IU/mL & $66.71 \pm 252.888$ & $62.84 \pm 260.328$ & $90.78 \pm 211.988$ & 0.010 & 0.24 \\
\hline CD4 count, cells/mL & $589.25 \pm 307.636$ & $610.30 \pm 308.668$ & $458.22 \pm 282.279$ & 0.000 & 0.47 \\
\hline APRI score $(<1 / \geq 1)$ & $48 / 26$ & $42 / 22$ & $6 / 4$ & 0.734 & - \\
\hline MELD score $(<10 / \geq 10)$ & $45 / 29$ & $40 / 24$ & $5 / 5$ & 0.500 & - \\
\hline CTP class (A/B) & $61 / 13$ & $53 / 11$ & $8 / 2$ & 0.561 & - \\
\hline Compensated cirrhosis (no/yes) & $64 / 10$ & $56 / 8$ & $8 / 2$ & 0.617 & - \\
\hline ALT, $\mu / \mathrm{L}$ & $53.28 \pm 42.374$ & $52.78 \pm 35.198$ & $56.50 \pm 76.668$ & 0.488 & - \\
\hline AST, $\mu / \mathrm{L}$ & $55.96 \pm 35.259$ & $54.61 \pm 30.681$ & $64.60 \pm 58.297$ & 0.567 & - \\
\hline $\operatorname{ALT}(<40 / \geq 40 \mu / \mathrm{L})$ & $32 / 42$ & $26 / 38$ & $6 / 4$ & 0.313 & - \\
\hline Hemoglobin, g/dL & $13.149 \pm 1.8851$ & $13.073 \pm 1.9490$ & $13.630 \pm 1.3913$ & 0.816 & - \\
\hline Platelets, $\mathrm{K} / \mu \mathrm{L}$ & $174.78 \pm 74.486$ & $178.56 \pm 73.534$ & $150.60 \pm 79.999$ & 0.301 & - \\
\hline
\end{tabular}

Data are presented as number or mean \pm SD.

SVR12, sustained virologic response at 12 weeks after treatment; HCV, hepatitis C virus; HIV, human immunodeficiency virus; W, white; B, black; A, Asian; H, Hispanic; O, others; BMI, body mass index; TN, treatment naïve; TE, treatment experienced; GT, genotype; APRI, aspartate aminotransferase-to-platelet ratio index; MELD, Model for End-Stage Liver Disease; CTP, Child-Turcotte-Pugh; ALT, alanine aminotransferase; AST, aspartate aminotransferase.

*Only variables with a p-value $<0.05$ in a univariate analysis were assessed.

fection. The regimen of DAAs varies based on the genotype of $\mathrm{HCV}$, prior treatment exposure, baseline NS5A resistant associated variants and the stage of liver fibrosis. ${ }^{19,20}$
In both major clinical trials and real-world data, DAAs have shown to have excellent response rate in HCV monoinfection. DAAs are also effective in HCV/HIV coinfection, and exist- 
Table 4. Characteristics of Patients Who Failed to Respond to Treatment

\begin{tabular}{|c|c|c|c|c|c|c|c|c|c|c|}
\hline No. & Regimen & Duration, wk & Failure type & Age, yr & Sex & Race & GT & $\mathrm{TN} / \mathrm{TE}$ & Cirrhosis & HIV status \\
\hline 1 & LDV/SOF & 12 & Relapse & 34 & $\mathrm{M}$ & Black & $1 \mathrm{a}$ & Naïve & No & Yes \\
\hline 2 & $\mathrm{LDV} / \mathrm{SOF}+\mathrm{RBV}$ & 24 & Relapse & 61 & $\mathrm{M}$ & Black & $1 \mathrm{~b}$ & $\mathrm{TE}$ & No & Yes \\
\hline 3 & LDV/SOF & 12 & Relapse & 58 & $\mathrm{M}$ & Black & $1 \mathrm{a}$ & $\mathrm{TE}$ & No & Yes \\
\hline 4 & $\mathrm{SOF} /+\mathrm{RBV}$ & 12 & Partial response & 65 & $\mathrm{M}$ & Hispanic & 2 & $\mathrm{TN}$ & Yes & No \\
\hline 5 & $\mathrm{SMV}+\mathrm{SOF}$ & 12 & Relapse & 58 & $\mathrm{M}$ & White & $1 \mathrm{a}$ & $\mathrm{TN}$ & Yes & No \\
\hline 6 & $\mathrm{SMV}+\mathrm{SOF}$ & 12 & Relapse & 67 & $\mathrm{M}$ & Black & $1 \mathrm{a}$ & $\mathrm{TN}$ & Yes & No \\
\hline 7 & $\mathrm{SMV}+\mathrm{SOF}$ & 12 & Relapse & 66 & $\mathrm{M}$ & Black & $1 \mathrm{a}$ & $\mathrm{TN}$ & Yes & No \\
\hline 8 & $\mathrm{SMV}+\mathrm{SOF}$ & 12 & Breakthrough & 60 & $\mathrm{M}$ & White & $1 \mathrm{a}$ & $\mathrm{TN}$ & Yes & Yes \\
\hline 9 & $\mathrm{SOF} /+\mathrm{RBV}$ & 24 & Relapse & 94 & $\mathrm{~F}$ & White & 3 & $\mathrm{TN}$ & Yes & No \\
\hline 10 & LDV/SOF & 12 & Partial response & 49 & $\mathrm{M}$ & Black & $1 \mathrm{a}$ & $\mathrm{TN}$ & No & Yes \\
\hline 11 & LDV/SOF & 12 & Relapse & 33 & $\mathrm{M}$ & Black & $1 \mathrm{a}$ & $\mathrm{TN}$ & No & No \\
\hline 12 & $\mathrm{SMV}+\mathrm{SOF}$ & 12 & Relapse & 64 & $\mathrm{M}$ & Other & 4 & $\mathrm{TN}$ & No & Yes \\
\hline 13 & $\mathrm{SMV}+\mathrm{SOF}$ & 12 & Relapse & 74 & M & Other & 4 & $\mathrm{TE}$ & No & Yes \\
\hline 14 & LDV/SOF & 12 & Partial response & 62 & $\mathrm{~F}$ & Black & $1 \mathrm{a}$ & $\mathrm{TN}$ & No & No \\
\hline 15 & $\mathrm{SOF} /+\mathrm{RBV}$ & 12 & Partial response & 67 & $\mathrm{~F}$ & Black & $1 \mathrm{a}$ & $\mathrm{TE}$ & No & No \\
\hline 16 & $\mathrm{LDV} / \mathrm{SOF}+\mathrm{RBV}$ & 24 & Relapse & 55 & $\mathrm{M}$ & Black & $1 \mathrm{a}$ & $\mathrm{TE}$ & Yes & Yes \\
\hline 17 & LDV/SOF & 12 & Partial response & 59 & $\mathrm{M}$ & Black & $1 \mathrm{a}$ & $\mathrm{TN}$ & No & Yes \\
\hline 18 & $\mathrm{OBV} / \mathrm{PTV} / \mathrm{r}+\mathrm{DSV}$ & 12 & Partial response & 66 & $\mathrm{M}$ & Black & $1 \mathrm{a}$ & $\mathrm{TN}$ & No & Yes \\
\hline 19 & LDV/SOF & 12 & Partial response & 60 & $\mathrm{~F}$ & Black & $1 \mathrm{a}$ & $\mathrm{TN}$ & No & No \\
\hline
\end{tabular}

GT, genotype; TN, treatment naïve; TE, treatment experienced; HIV, human immunodeficiency virus; M, male; F, female; LDV, ledipasvir; SOF, sofosbuvir; RBV, ribavirin; SMV, simeprevir; OBV, ombitasvir; PTV/r, paritaprevir+ritonavir; DSV, dasabuvir.

Table 5. Adverse Events Associated with Treatment Regimens

\begin{tabular}{lcccc}
\hline & & \multicolumn{2}{c}{ HIV status } & \\
\cline { 3 - 4 } $\begin{array}{l}\text { Adverse } \\
\text { events }\end{array}$ & $\begin{array}{c}\text { Total } \\
(\mathrm{n}=327)\end{array}$ & $\begin{array}{c}\text { Negative } \\
(\mathrm{n}=253)\end{array}$ & $\begin{array}{c}\text { Positive } \\
(\mathrm{n}=74)\end{array}$ & p-value \\
\hline Fatigue & $89(27.2)$ & $65(25.6)$ & $24(32.4)$ & 0.298 \\
Insomnia & $4(1.2)$ & $4(1.5)$ & $0(0)$ & 0.578 \\
Headache & $16(4.8)$ & $13(5.1)$ & $3(4)$ & 0.704 \\
Nausea & $15(4.5)$ & $11(4.3)$ & $4(5.4)$ & 0.752 \\
Vomiting & $2(0.6)$ & $0(0)$ & $2(2.7)$ & 0.051 \\
Diarrhea & $1(0.3)$ & $1(0.3)$ & $0(0)$ & 0.588 \\
Constipation & $2(0.6)$ & $2(0.7)$ & $0(0)$ & 0.443 \\
Abdominal & $5(1.5)$ & $1(0.3)$ & $4(5.4)$ & 0.010 \\
pain & & & & \\
Rash & $16(4.9)$ & $12(4.7)$ & $4(5.4)$ & 0.765 \\
Arthralgia & $14(4.2)$ & $10(3.9)$ & $4(5.4)$ & 0.529 \\
Anemia & $47(14.3)$ & $35(13.8)$ & $12(16.2)$ & 0.577 \\
Thrombocy- & $14(4.2)$ & $8(3.1)$ & $6(8)$ & 0.095 \\
topenia & & & & \\
Leucopenia & $35(10.7)$ & $20(7.9)$ & $15(20.2)$ & 0.005 \\
Itching & $7(2.1)$ & $4(1.5)$ & $3(4)$ & 0.194 \\
Dizziness & $8(2.4)$ & $5(1.9)$ & $3(4)$ & 0.387 \\
Photosensi- & $4(1.2)$ & $2(0.7)$ & $2(2.7)$ & 0.222 \\
tive rash & & & & \\
\hline
\end{tabular}

Data are presented as number (\%).

HIV, human immunodeficiency virus. ing studies have shown similar response rate between HCV monoinfection and HCV/HIV coinfection group. However, data regarding the response of DAAs in real-world setting HCV/HIV coinfection is limited. In our study, patients with HCV monoinfection had a statistically significant higher virologic response than those with HCV/HIV coinfection. The response rate was similar across groups receiving different antiviral regimens, and SVR12 did not vary based on genotype. The SVR12 achieved $(96 \%)$ in our study is similar to most other studies in case of HCV monoinfection but a slight decline of response rate (86\%) observed in HCV/HIV coinfection group. ${ }^{23-27}$

Factors associated with lower SVR12 were identified with univariate analysis and validity was verified by multivariate analysis by adjusting variables. One of the variables evaluated was pretreatment HCV RNA and it was not identified as a predictor of treatment response, consistent with most of the study findings. Rivero-Juarez et al. ${ }^{28}$ and Rallón et al. ${ }^{29}$ concluded that pretreatment HCV RNA viral load was significantly associated with SVR in coinfected patients treated with pegylated interferon and ribavirin and that HCV viral load $>600,000 \mathrm{IU} / \mathrm{mL}$ was a predictor of relapse. However, with current DAAs treatment regimens, pretreatment HCV RNA levels have limited value, mostly used to choose the duration of treatment and have not been shown as a predictor of treatment failure in coinfected patients. ${ }^{23,30,31}$ Most literature which assessed DAAs response in $\mathrm{HCV} / \mathrm{HIV}$ coinfection did not find a significant difference in 
response rates between monoinfections versus coinfections, and also did not identify HIV coinfection as a predictor of treatment response. ${ }^{23,30}$ However, one large multi-cohort prospective study published recently from Spain by Neukam et al. ${ }^{32}$ showed that HIV coinfected patients had higher rates of relapse leading to significantly lower response rates compared to monoinfected patients (86.3\% vs 94.9\% respectively, $\mathrm{p}=0.002$ ). There was no identifiable relationship with HIV viral load and CD4 number with treatment response or as a predictor of relapse in that study. Similar results were reflected in our study where despite a difference in SVR12 rates in mono and coinfected group, high HIV viremia and lower CD4 counts in the coinfected group were not associated with lower SVR. The trend towards treatment failure should be assessed in future studies with a larger cohort coinfection cohort, as genetic factors, length of HIV infection, and drug resistance may also be variables in attenuated response in coinfections. Additionally, the larger cohort may clarify the associations of liver injury in coinfections and identify them as predictors.

The results demonstrated in our study is based on the realworld setting, which differs from most other literature. The advantage of real-world setting is that a small clinical trial with strict protocols may overlook real-world factors of response, including compliance, individual patient characteristics, and nonhomogenous clinical management. These variations may help explain the difference in response rates between our study and existing literature.

More than three-fifths of our study patients were black, but there was no difference in response rate noted based on race. This varies from some studies in literature, where the response was lower in the black population compared to non-black in HCV monoinfection. ${ }^{33-36}$ Pre-treatment platelet count was found to be a strong predictor of overall SVR, which is consistent with some pre-existing literature (Lawitz et al., 2016). ${ }^{37}$ Patient who achieved SVR12 had higher mean baseline platelet compared to those who did not achieve SVR12 (193 K/ $\mathrm{LL}$ vs $141 \mathrm{~K}$ ) $\mu \mathrm{L}, \mathrm{p}=0.002$ ), which may be attributable to low platelets as a marker of portal hypertension, and its relationship to advanced fibrosis and cirrhosis. ${ }^{38}$ We noted that compensated cirrhosis was not identified as a predictor of treatment response similar to some other studies in the literature. ${ }^{25,39}$ However, in both HCV monoinfected and HCV/HIV coinfected groups in our study, advanced compensated cirrhosis or CTP class B cirrhosis was significantly associated with lower treatment response ( $p=0.013$ ). As a result, special consideration in choosing therapy may be required in advanced cirrhosis or CTP class B cirrhosis.

Tolerability and safety of DAAs are presumed to be an issue in the coinfected cohort because of drug interactions. Abdominal pain and leucopenia were observed more in the coinfected group than monoinfected may reflect drug interaction but none were significant and severe enough leading to discontinuation. Cause of leucopenia observed in the coinfected group is very difficult to identify due to a wide range of issue related to leucopenia. It could be due to HIV disease itself, or HIV medication or interaction between HIV medication and DAAs. Tolerability was excellent and no patient even needed major dose adjustment during the study period. An experienced HIV specialist of the same center assisted in choosing treatment resulting in a well-balanced regimen with less drug interaction and fewer side effects, which may help explain the lack of significant adverse events. Antiretroviral regimens used in our cohort were lamivudine, raltegravir, ritonavir, darunavir, zidovudine, efavirenz, cobicistat, abacavir, lopinavir, emtricitabine, tenofovir, rilpivirine, and dolutegravir.

However, our study was unique in assessing and comparing the real-world effectiveness, tolerability and safety of different therapeutic regimens in HCV monoinfection as well as HCV/HIV coinfection. Our study also incorporated a substantial number of black patients who are historically regarded as the difficult to treat population and shown to have lower response. ${ }^{33-36}$ One other strength of our study is the representation of a significant number of patients with HCV genotype 4 in contrast to available literature where genotype 4 outcomes are rarely reported due to poor representation.

Limitations of our study include using a retrospective design, a small number of HCV/HIV coinfected patients, insufficient documentation of adverse effects and lack of viral resistance testing. Additionally, the number of patients in some treatment regimens or genotypes was too small for meaningful conclusions.

In the real-world setting, interferon-free direct acting antiviral regimens may have a significantly lower virological response in HCV/HIV coinfection compared to HCV monoinfection. Treatment in HCV/HIV coinfected group needs particular attention while choosing DAA regimen and duration of treatment. Treatment appears to be safe in both coinfection and monoinfections, as no major adverse effects and drug interactions lead to discontinuation and relapse.

\section{CONFLICTS OF INTEREST}

No potential conflict of interest relevant to this article was reported.

\section{REFERENCES}

1. Balogun MA, Ramsay ME, Hesketh LM, et al. The prevalence of hepatitis C in England and Wales. J Infect 2002;45:219-226.

2. Mocroft A, Katlama C, Johnson AM, et al. AIDS across Europe, 1994-98: the EuroSIDA study. Lancet 2000;356:291-296.

3. Kirk GD, Mehta SH, Astemborski J, et al. HIV, age, and the severity of hepatitis $C$ virus-related liver disease: a cohort study. Ann Intern Med 2013;158:658-666.

4. Goedert JJ, Eyster ME, Lederman MM, et al. End-stage liver dis- 
ease in persons with hemophilia and transfusion-associated infections. Blood 2002;100:1584-1589.

5. Vogel M, Page E, Boesecke C, et al. Liver fibrosis progression after acute hepatitis $\mathrm{C}$ virus infection in HIV-positive individuals. Clin Infect Dis 2012;54:556-559.

6. Soriano V, Labarga P, Ruiz-Sancho A, Garcia-Samaniego J, Barreiro P. Regression of liver fibrosis in hepatitis C virus/HIV-coinfected patients after treatment with pegylated interferon plus ribavirin. AIDS 2006;20:2225-2227.

7. Hepatitis C Working Group for the Collaboration of Observational HIV Epidemiological Research Europe (COHERE) in EuroCoord. Is response to anti-hepatitis $\mathrm{C}$ virus treatment predictive of mortality in hepatitis C virus/HIV-positive patients? AIDS 2017;31:661-668.

8. Berenguer J, Zamora FX, Carrero A, et al. Effects of sustained viral response in patients with HIV and chronic hepatitis $\mathrm{C}$ and nonadvanced liver fibrosis. J Acquir Immune Defic Syndr 2014;66:280287.

9. Labarga P, Soriano V, Vispo ME, et al. Hepatotoxicity of antiretroviral drugs is reduced after successful treatment of chronic hepatitis C in HIV-infected patients. J Infect Dis 2007;196:670-676.

10. Martin TC, Martin NK, Hickman M, et al. Hepatitis C virus reinfection incidence and treatment outcome among HIV-positive MSM. AIDS 2013;27:2551-2557.

11. Hulskotte EG, Feng HP, Xuan F, et al. Pharmacokinetic interactions between the hepatitis $\mathrm{C}$ virus protease inhibitor boceprevir and ritonavir-boosted HIV-1 protease inhibitors atazanavir, darunavir, and lopinavir. Clin Infect Dis 2013;56:718-726.

12. Torriani FJ, Rodriguez-Torres M, Rockstroh JK, et al. Peginterferon Alfa-2a plus ribavirin for chronic hepatitis $\mathrm{C}$ virus infection in HIV-infected patients. N Engl J Med 2004;351:438-450.

13. Sulkowski MS, Naggie S, Lalezari J, et al. Sofosbuvir and ribavirin for hepatitis C in patients with HIV coinfection. JAMA 2014;312: 353-361.

14. Osinusi A, Townsend K, Kohli A, et al. Virologic response following combined ledipasvir and sofosbuvir administration in patients with HCV genotype 1 and HIV co-infection. JAMA 2015;313: 1232-1239.

15. Saeed S, Strumpf EC, Walmsley SL, et al. How generalizable are the results from trials of direct antiviral agents to people coinfected with HIV/HCV in the real world? Clin Infect Dis 2016;62:919926.

16. Bhattacharya D, Belperio PS, Shahoumian TA, et al. Effectiveness of all-oral antiviral regimens in 996 human immunodeficiency virus/hepatitis C virus genotype 1-coinfected patients treated in routine practice. Clin Infect Dis 2017;64:1711-1720.

17. Khatri A, Dutta S, Wang H, et al. Evaluation of drug-drug interactions between hepatitis $\mathrm{C}$ antiviral agents ombitasvir, paritaprevir/ ritonavir, and dasabuvir and HIV-1 protease inhibitors. Clin Infect Dis 2016;62:972-979.

18. Wyles D, Saag M, Viani RM, et al. TURQUOISE-I Part 1b: ombitasvir/paritaprevir/ritonavir and dasabuvir with ribavirin for hepatitis C virus infection in HIV-1 coinfected patients on darunavir. J In- fect Dis 2017:215:599-605.

19. European Association for Study of Liver. EASL recommendations on treatment of hepatitis C 2015. J Hepatol 2015;63:199-236.

20. AASLD/IDSA HCV Guidance Panel. Hepatitis C guidance: AASLDIDSA recommendations for testing, managing, and treating adults infected with hepatitis C virus. Hepatology 2015;62:932-954.

21. Poordad F, McCone J Jr, Bacon BR, et al. Boceprevir for untreated chronic HCV genotype 1 infection. N Engl J Med 2011;364:11951206.

22. Jacobson IM, McHutchison JG, Dusheiko G, et al. Telaprevir for previously untreated chronic hepatitis C virus infection. N Engl J Med 2011;364:2405-2416.

23. Milazzo L, Lai A, Calvi E, et al. Direct-acting antivirals in hepatitis C virus (HCV)-infected and HCV/HIV-coinfected patients: real-life safety and efficacy. HIV Med 2017;18:284-291.

24. Afdhal N, Zeuzem S, Kwo P, et al. Ledipasvir and sofosbuvir for untreated HCV genotype 1 infection. N Engl J Med 2014; 370:1889-1898.

25. Afdhal N, Reddy KR, Nelson DR, et al. Ledipasvir and sofosbuvir for previously treated HCV genotype 1 infection. N Engl J Med 2014;370:1483-1493.

26. Ferenci P, Bernstein D, Lalezari J, et al. ABT-450/r-ombitasvir and dasabuvir with or without ribavirin for HCV. N Engl J Med 2014; 370:1983-1992.

27. Lawitz E, Sulkowski MS, Ghalib R, et al. Simeprevir plus sofosbuvir, with or without ribavirin, to treat chronic infection with hepatitis $\mathrm{C}$ virus genotype 1 in non-responders to pegylated interferon and ribavirin and treatment-naïve patients: the COSMOS randomised study. Lancet 2014;384:1756-1765.

28. Rivero-Juarez A, Mira JA, Camacho A, et al. Baseline risk factors for relapse in HIV/HCV co-infected patients treated with PEG-IFN/ RBV. Infection 2013;41:21-26.

29. Rallón NI, Naggie S, Benito JM, et al. Association of a single nucleotide polymorphism near the interleukin-28B gene with response to hepatitis C therapy in HIV/hepatitis C virus-coinfected patients. AIDS 2010;24:F23-F29.

30. Bruno G, Saracino A, Scudeller L, et al. HCV mono-infected and $\mathrm{HIV} / \mathrm{HCV}$ co-infected individuals treated with direct-acting antivirals: to what extent do they differ? Int J Infect Dis 2017;62:64-71.

31. Werner CR, Schwarz JM, Egetemeyr DP, et al. Second-generation direct-acting-antiviral hepatitis C virus treatment: efficacy, safety, and predictors of SVR12. World J Gastroenterol 2016;22:80508059.

32. Neukam K, Morano-Amado LE, Rivero-Juárez A, et al. HIVcoinfected patients respond worse to direct-acting antiviralbased therapy against chronic hepatitis $\mathrm{C}$ in real life than HCVmonoinfected individuals: a prospective cohort study. HIV Clin Trials 2017;18:126-134.

33. Naggie S, Cooper C, Saag M, et al. Ledipasvir and sofosbuvir for HCV inpatients coinfected with HIV-1. N Engl J Med 2015;373: 705-713.

34. Lawitz E, Poordad FF, Pang PS, et al. Sofosbuvir and ledipasvir 
fixed-dose combination with and without ribavirin in treatmentnaive and previously treated patients with genotype 1 hepatitis $C$ virus infection (LONESTAR): an open-label, randomised, phase 2 trial. Lancet 2014;383:515-523.

35. Jansen JW, Powderly GM, Linneman TW. Identification of predictors for treatment failure in hepatitis $\mathrm{C}$ virus patients treated with ledipasvir and sofosbuvir. Ann Pharmacother 2017;51:543-547.

36. Cavalcante LN, Lyra AC. Predictive factors associated with hepatitis C antiviral therapy response. World J Hepatol 2015;7:16171631.

37. Lawitz E, Matusow G, DeJesus E, et al. Simeprevir plus sofosbuvir in patients with chronic hepatitis C virus genotype 1 infection and cirrhosis: a phase 3 study (OPTIMIST-2). Hepatology 2016;64:360369.

38. Marot A, Trépo E, Doerig C, Schoepfer A, Moreno C, Deltenre P. Liver stiffness and platelet count for identifying patients with compensated liver disease at low risk of variceal bleeding. Liver Int 2017;37:707-716.

39. Leroy V, Angus P, Bronowicki JP, et al. Daclatasvir, sofosbuvir, and ribavirin for hepatitis $\mathrm{C}$ virus genotype 3 and advanced liver disease: a randomized phase III study (ALLY-3+). Hepatology 2016;63:1430-1441. 